\title{
Establishment and Validation of a Nomographic Model for Individualized Prediction of Invasive Pulmonary Fungal Infection after Chemotherapy for Hematologic Tumor
}

\author{
LING LIU AND SHIHAI ZHENG* \\ Department of Medical Laboratory, The First People's Hospital of Chongqing Liang Jiang New Area, Chongqing 401121, \\ China
}

Liu et al.: Nomographic Model for Invasive Pulmonary Fungal Infection after Chemotherapy

To retrospectively analyze the risk factors of invasive pulmonary fungal infection after chemotherapy in patients with hematologic tumor and to establish and validate related nomographic models. 368 patients with hematologic tumor who were treated with chemotherapy were collected. The influencing factors of invasive pulmonary fungal infection after chemotherapy were analyzed by univariate and multivariate logistic regression. Based on the analyzed risk factors, the nomogram prediction model was established and verified internally. Of the 368 patients with hematologic tumor, $35.33 \%(130 / 368)$ had fungal infection after chemotherapy and $64.67 \%(238 / 368)$ had non-fungal infection. Univariate and multivariate logistic regression analysis showed that hypoproteinemia (Odds ratio=3.220,95 \% confidence interval: 1.886 5.496), broad-spectrum antibiotic use $\geq 2$ kinds (Odds ratio=2.616, $95 \%$ confidence interval: $1.514 \sim 4.518$ ), broadspectrum antibiotic duration $\geq 7 \mathrm{~d}$ (Odds ratio=2.444, $95 \%$ confidence interval: 1.455 4.103), duration of agranulocytosis $\geq 7 \mathrm{~d}$ (Odds ratio=3.112, $95 \%$ confidence interval: $1.804 \sim 5.368)$, cytomegalovirus or Epstein-Barr virus infection (Odds ratio $=6.170,95 \%$ confidence interval: $3.304 \sim 11.524)$ were risk factors for fungal infection after chemotherapy in patients with hematologic tumors. Based on the above five risk factors, the prediction model of nomogram was established and verified by Bootstrap method for 500 times. The calibration curve fitted well with the standard curve. The receiver operating characteristic curve area under the curve was $0.762,95 \%$ confidence interval: $0.7095-0.8146$ and the Hosmer-Lemeshow test $\mathbf{p}>\mathbf{0 . 0 5}$, which indicated that the prediction model of nomogram had good discrimination and accuracy. Hypoproteinemia, broad-spectrum antibiotic use $\geq 2$ kinds, broad-spectrum antibiotic duration $\geq 7 \mathrm{~d}$, duration of agranulocytosis $\geq 7 \mathrm{~d}$ and cytomegalovirus or Epstein-Barr virus infection are the risk factors of concurrent fungal infection after chemotherapy in patients with hematologic tumor. After verification, the constructed nomogram has high discrimination and accuracy, which has dual guiding significance for clinical screening of high-risk population and making individualized prevention and treatment measures.

Key words: Hematologic tumor, chemotherapy, invasive pulmonary fungal infection, nomographic model

Hematological malignant tumors are a group of hematopoietic diseases including leukemia, lymphoma and multiple myeloma, which are characterized by high degree of malignancy, complex treatment and poor prognosis $^{[1]}$. Unlike solid tumors, hematologic tumors are often treated with chemoradiotherapy combined with bone marrow transplantation. However, during chemotherapy, the patient's white blood cell count will decrease and immune function will decrease, which is very easy to cause infection. The most common infection is pulmonary infection, which seriously threatens the patient's life safety and the patient's therapeutic effect $^{[2]}$. Several clinical studies have evaluated the benefit of fungal prophylaxis during chemotherapy and hematopoietic stem cell transplantation in patients with hematologic malignant tumors and have shown an improvement in the incidence of invasive pulmonary fungal infections and associated mortality ${ }^{[3,4]}$. The premise of preventive treatment measures is how to better predict the occurrence risk, especially to identify

*Address for correspondence

E-mail: liuling0710dym@163.com 
the population with high risk of infection. Therefore, this study retrospectively analyzed the clinical data of 368 patients with hematologic tumor chemotherapy, probed into the risk factors of invasive pulmonary fungal infection and established nomogram prediction model, which provided reference value for early diagnosis and early intervention.

\section{MATERIALS AND METHODS}

\section{Study objects:}

From January 2018 to January 2021, the clinical data of 368 patients with hematological tumors treated with in-patient chemotherapy in 5 hospitals of Class III and Grade A in urban areas were collected. Inclusion criteria: Hematologic tumor meets relevant diagnostic criteria in "Response and diagnostic criteria for hematologic diseases"[5]; "Diagnostic criteria and therapeutic principles for invasive fungal infection in patients with hematologic diseases/malignant tumors"[6]; patients and their families are informed about the study and sign the informed consent form. Exclusion criteria: Patients with repeated admission and admission; patients with other critical diseases, such as other malignant tumors, liver cirrhosis and coronary heart disease; patients mainly treated with transplantation; patients with fungal infection at admission or $48 \mathrm{~h}$ after admission. This study has been approved by the ethics committee.

\section{Criteria for diagnosis:}

Hematologic malignancies: Refer to the relevant diagnostic criteria in "Response and Diagnostic Criteria for Hematologic Diseases"[5] compiled by Shen and Zhao et al. to diagnose hematologic tumors, such as leukemia, multiple myeloma, Hodgkin's lymphoma and non-Hodgkin's lymphoma.

Invasive fungal disease: The diagnosis of fungal infection is divided into three levels and the diagnostic criteria are as follows; The diagnosis: There are predisposing factors for fungal infection, the imaging examination results are sufficient to confirm the occurrence of deep tissue infection, the blood sample culture is positive and there are clinical signs of pathogenic infection; The clinical diagnosis: There are predisposing factors for fungal infection, the chest Computed Tomography (CT) imaging shows halo sign or crescent air sign, or the lesion site presents empty cavity sign, clinical symptoms and signs of lung tissue infection; The possible diagnosis: probable factors of fungal infection, clinical signs of pathogenic infection, fever lasting for more than $4 \mathrm{~d}$, ineffective treatment with broad-spectrum antibiotics.

\section{Study methods:}

This study used a retrospective analysis method to collect general data of all patients, including age, gender, hospital stay, presence or absence of diseases and occupations such as diabetes and hypertension; clinical data, including whether there was remission of primary disease, presence or absence of Peripherally Inserted Central Catheter (PICC), presence or absence of hypoproteinemia, presence or absence of hematopoietic stem cell transplantation, presence or absence of bacterial infection, duration of hormone use, type of broad-spectrum antibiotic use and duration of continuous use, duration of agranulocytosis, the presence or absence of immunosuppressive agents and the presence or absence of cytomegalovirus or EpsteinBarr virus infection were proposed as contributing factors.

\section{Statistical analysis:}

Statistical Package for the Social Sciences (SPSS) 25.0 software was used to process the data of this study. Quantitative data conforming to normal distribution were expressed as $(\overline{\mathrm{x}} \pm \mathrm{s}), \mathrm{t}$ test was used for comparison between the two groups. Analysis of variance was used for comparison between the two groups. Categorical data were expressed as $n(\%) \cdot \chi^{2}$ test was performed between the two groups. Univariate and multivariate logistic regression analysis was used to screen the independent influencing factors of invasive pulmonary fungal infection after chemotherapy in patients with hematologic tumors. The Regression Modeling Strategies (RMS) package in R language (R4.0.5) was used to establish the nomographic model of the risk of invasive pulmonary fungal infection and 500 times of sampling were repeated by Bootstrap method for internal verification; the Hosmer-Lemeshow goodnessof-fit test was used, when $\mathrm{p}>0.05$, it was considered that the goodness-of-fit of the model was good; the Receiver Operating Characteristic (ROC) curve was used to determine the cut-off point of diagnosis and to evaluate the predictive effect of the model. Test level Alpha $(\alpha)=0.05$.

\section{RESULTS AND DISCUSSION}

Among 368 patients with hematological tumors enrolled, 130 patients were complicated with invasive pulmonary fungal infection, accounting for $35.33 \%$; the distribution of primary disease and 130 patients with Invasive Fungal Diseases (IFD) after chemotherapy in 368 patients is shown in Table 1 and Table 2. 
TABLE 1: DISTRIBUTION OF PRIMARY DISEASE IN ALL CASES [n (\%)]

\begin{tabular}{lcc}
\hline Primary disease & $\mathbf{n}$ & $\mathrm{n}(\%)$ \\
\hline Acute myeloid leukemia & 103 & $27.99 \%$ \\
Multiple myeloma & 59 & $16.03 \%$ \\
Myelodysplastic syndrome & 44 & $11.96 \%$ \\
Acute lymphoblastic leukemia & 30 & $8.15 \%$ \\
Hodgkin's lymphoma & 13 & $3.53 \%$ \\
Non-Hodgkin's lymphoma & 70 & $19.02 \%$ \\
Chronic granulocytic leukemia & 8 & $2.17 \%$ \\
(accelerated phase) & 41 & $11.14 \%$ \\
Other & 368 & $100 \%$ \\
\hline
\end{tabular}

TABLE 2: DISTRIBUTION OF ALL PRIMARY CASES WITH CONCOMITANT IFD [n (\%)]

\begin{tabular}{lcc}
\hline Primary disease & Concomitant IFD & $\mathrm{n}(\%)$ \\
\hline Acute myeloid leukemia & 37 & $28.46 \%$ \\
Multiple myeloma & 20 & $15.38 \%$ \\
Myelodysplastic syndrome & 23 & $17.69 \%$ \\
Acute lymphoblastic leukemia & 3 & $2.31 \%$ \\
Acute promyelocytic leukemia & 4 & $3.08 \%$ \\
Non-Hodgkin's lymphoma & 17 & $13.08 \%$ \\
Chronic granulocytic leukemia & 7 & $5.38 \%$ \\
(accelerated phase) & 19 & $14.62 \%$ \\
Other & 130 & $100 \%$ \\
Total & & \\
\hline
\end{tabular}

Note: IFD: Invasive Fungal Diseases

Univariate analysis of IFD in patients with hematologic tumors after chemotherapy was shown below. Among 368 patients with hematologic tumors in this study, $35.33 \%(130 / 368)$ had IFD and $64.67 \%(238 / 368)$ had non-IFD after chemotherapy. In univariate analysis, there were significant differences in gender, presence or absence of hypoproteinemia, type of broad-spectrum antibiotic use, duration of broad-spectrum antibiotic, duration of agranulocytosis, presence or absence of cytomegalovirus or Epstein-Barr virus infection between the two groups (all $\mathrm{p}<0.05)$ (Table 3 ).

Multivariate logistic analysis was performed to determine whether IFD $(0=$ non-IFD, $1=$ IFD) was complicated ornot. Theresults showed:hypoproteinemia (Odds Ratio $(\mathrm{OR})=3.220,95 \%$ Confidence Interval (CI): 1.886 5.496), broad-spectrum antibiotic use $\geq 2$ kinds $(\mathrm{OR}=2.616,95 \% \mathrm{CI}: 1.514 \sim 4.518)$, broadspectrum antibiotic duration $\geq 7 \mathrm{~d}(\mathrm{OR}=2.444,95 \%$ CI: 1.455 4.103), duration of agranulocytosis $>7 \mathrm{~d}$ $(\mathrm{OR}=3.112,95 \% \mathrm{CI}: 1.804 \sim 5.368)$, cytomegalovirus or Epstein-Barr virus infection $(\mathrm{OR}=6.170,95 \%$ CI: 3.304 11.524) were risk factors for IFD after chemotherapy in patients with hematologic tumors $(\mathrm{p}<0.05)$ (Table 4 and Table 5).

Establishment and evaluation of nomographic model of IFD in patients with hematologic tumors after chemotherapy was described here. A nomographic model was constructed based on multivariate unconditional logistic regression for risk factors of hypoproteinemia, broad-spectrum antibiotic use $\geq 2$ kinds, duration of broad-spectrum antibiotic $\geq 7 \mathrm{~d}$, duration of agranulocytosis $>7 \mathrm{~d}$, cytomegalovirus or Epstein-Barr virus infection for IFD after chemotherapy in patients with hematologic tumors (fig. 1). After repeated sampling for 500 times by Bootstrap method for internal validation, the calibration curves and standard curve fitting were all good (fig. 2); ROC curve Area Under the Curve (AUC) $=0.762,95 \% \mathrm{CI}$ : $0.7095 \sim 0.8146$, indicating that this model has good prediction accuracy (fig. 3 ), $p=0.200>0.05$ by HosmerLemeshow goodness-of-fit test, indicating that the difference between the model predicted value and the actual value was not statistically significant (fig. 1-fig. $3)$. 
TABLE 3: UNIVARIATE ANALYSIS OF IFD IN PATIENTS WITH HEMATOLOGIC TUMORS AFTER CHEMOTHERAPY [n (\%)]

\begin{tabular}{|c|c|c|c|c|}
\hline Contents & Non-IFD $(n=238)$ & IFD $(n=130)$ & $\chi^{2}$ & $\mathrm{p}$ \\
\hline Age (years old) & & & 4.018 & 0.029 \\
\hline$<60$ & $134(56.30)$ & $59(45.38)$ & & \\
\hline$\geq 60$ & $104(43.70)$ & $71(54.62)$ & & \\
\hline Gender & & & 0.927 & 0.197 \\
\hline Male & $112(47.06)$ & $68(52.31)$ & & \\
\hline Female & $126(52.94)$ & $62(47.69)$ & & \\
\hline Hospital stay (d) & & & 0.305 & 0.331 \\
\hline$<2$ & $93(39.08)$ & $47(36.15)$ & & \\
\hline$\geq 2$ & $145(60.92)$ & $83(63.85)$ & & \\
\hline Concurrent diabetes & & & 2.315 & 0.079 \\
\hline No & $113(47.48)$ & $51(39.23)$ & & \\
\hline Yes & $125(52.52)$ & $79(60.77)$ & & \\
\hline $\begin{array}{l}\text { Concurrent } \\
\text { hypertension }\end{array}$ & & & 1.592 & 0.125 \\
\hline No & $104(43.70)$ & $48(36.92)$ & & \\
\hline Yes & $134(56.30)$ & $82(63.08)$ & & \\
\hline Occupation & & & 0.196 & 0.658 \\
\hline Public institution & $49(20.59)$ & $28(21.54)$ & & \\
\hline Worker/peasant & 97 (40.76) & $61(46.92)$ & & \\
\hline Self-employment & $63(26.47)$ & $16(12.31)$ & & \\
\hline Other & $29(12.18)$ & $25(19.23)$ & & \\
\hline $\begin{array}{l}\text { Primary disease } \\
\text { remission }\end{array}$ & & & 0.852 & 0.208 \\
\hline No & 89 (37.39) & $55(42.31)$ & & \\
\hline Yes & $149(62.61)$ & 75 (57.69) & & \\
\hline PICC catheter & & & 1.932 & 0.102 \\
\hline No & $71(29.83)$ & $48(36.92)$ & & \\
\hline Yes & $167(70.17)$ & $82(63.08)$ & & \\
\hline Hypoproteinemia & & & 5.477 & 0.012 \\
\hline No & $87(36.55)$ & $32(24.62)$ & & \\
\hline Yes & $151(63.44)$ & $98(75.38)$ & & \\
\hline $\begin{array}{l}\text { Hematopoietic stem } \\
\text { cell transplantation }\end{array}$ & & & 0.057 & 0.456 \\
\hline No & $65(27.31)$ & $34(26.15)$ & & \\
\hline Yes & $173(72.69)$ & $96(73.85)$ & & \\
\hline $\begin{array}{l}\text { Co-infection with } \\
\text { bacteria }\end{array}$ & & & 3.011 & 0.053 \\
\hline No & $67(28.15)$ & $48(36.92)$ & & \\
\hline Yes & $171(71.85)$ & $82(63.08)$ & & \\
\hline $\begin{array}{l}\text { Duration of hormone } \\
\text { use (d) }\end{array}$ & & & 0.428 & 0.294 \\
\hline
\end{tabular}


$<5$

$\geq 5$

Broad-spectrum

antibiotic use

$<2$ kinds

$\geq 2$ kinds

Broad spectrum antibiotic duration (d)

$<7$

$\geq 7$

Duration of agranulocytosis

$<7$

$\geq 7$

With or without immunosuppressant use

No

Yes

Cytomegalovirus or Epstein-Barr virus infection

No

Yes

$$
78 \text { (32.77) }
$$

$160(67.23)$

47 (36.15)

$83(63.85)$

0.012

89 (37.39)

33 (25.38)

149 (62.61)

97 (74.62)

4.502

0.024

48 (20.17)

39 (30.00)

190 (79.83)

91 (70.00)

3.323

0.043

98 (41.18)

41 (31.54)

140 (58.82)

89 (68.46)

0.560

0.264

68 (28.57)

42 (32.31)

170 (71.43)

88 (67.69)

Note: Hypoproteinemia-Plasma albumin $\leq 30 \mathrm{~g} / \mathrm{l}$; Hormone duration-Data within $14 \mathrm{~d}$ before infection for patients with IFD and longest cumulative duration within $14 \mathrm{~d}$ for patients without IFD; Broad-spectrum antibiotic use-Data for patients with IFD, take broad-spectrum antibiotic use within $14 \mathrm{~d}$ before infection and for patients without IFD, take data of accumulative broad-spectrum antibiotic use within 14 d; Broad-spectrum antibiotic duration-For patients with IFD, take data within $14 \mathrm{~d}$ before infection and for patients without IFD, take data of longest time of accumulative use within $14 \mathrm{~d}$; Duration of agranulocytosis-For patients with IFD, take the last agranulocytosis days before infection and for patients with non-IFD, take the longest day of agranulocytes, where IFD: Invasive Fungal Diseases

TABLE 4: DESCRIPTION OF ASSIGNMENT

\begin{tabular}{lcc}
\hline Variable & Code & Assignment \\
\hline Age (y old) & X1 & $0 \leq 60,1 \geq 60$ \\
Hypoproteinemia & X2 & $0=$ Absent, 1=present \\
Broad-spectrum antibiotic use & X3 & $0 \leq 2,1 \geq 2$ \\
Broad spectrum antibiotic duration & X4 & $0 \leq 7,1 \geq 7$ \\
Duration of agranulocytosis & X5 & $0 \leq 7,1 \geq 7$ \\
Cytomegalovirus or Epstein-Barr virus & X6 & $0=$ Absent, $1=$ present \\
infection & Y & $0=$ Non-IFD, 1=IFD \\
Concurrent IFD &
\end{tabular}

TABLE 5: MULTIVARIATE LOGISTIC ANALYSIS OF IFD IN PATIENTS WITH HEMATOLOGIC TUMORS AFTER CHEMOTHERAPY

\begin{tabular}{|c|c|c|c|c|c|c|}
\hline Factor & Beta (B) & Standard Error (SE) & Wald & $\mathrm{p}$ & OR & $95 \% \mathrm{Cl}$ \\
\hline Constant term & -4.724 & 0.603 & 61.323 & $<0.001$ & - & - \\
\hline Hypoproteinemia & 1.169 & 0.273 & 18.370 & $<0.001$ & 3.220 & $1.886 \sim 5.496$ \\
\hline $\begin{array}{l}\text { Broad spectrum antibiotic } \\
\text { use } \geq 2 \text { kinds }\end{array}$ & 0.962 & 0.279 & 11.889 & 0.001 & 2.616 & $1.514 \sim 4.518$ \\
\hline $\begin{array}{l}\text { Broad spectrum antibiotic } \\
\text { duration } \geq 7 \mathrm{~d}\end{array}$ & 0.893 & 0.264 & 11.422 & 0.001 & 2.444 & $1.455 \sim 4.103$ \\
\hline
\end{tabular}


Duration of agranulocytosis

$>7 \mathrm{~d}$

Cytomegalovirus or Epstein-

Barr virus infection
1.135

0.278

1.820

0.319

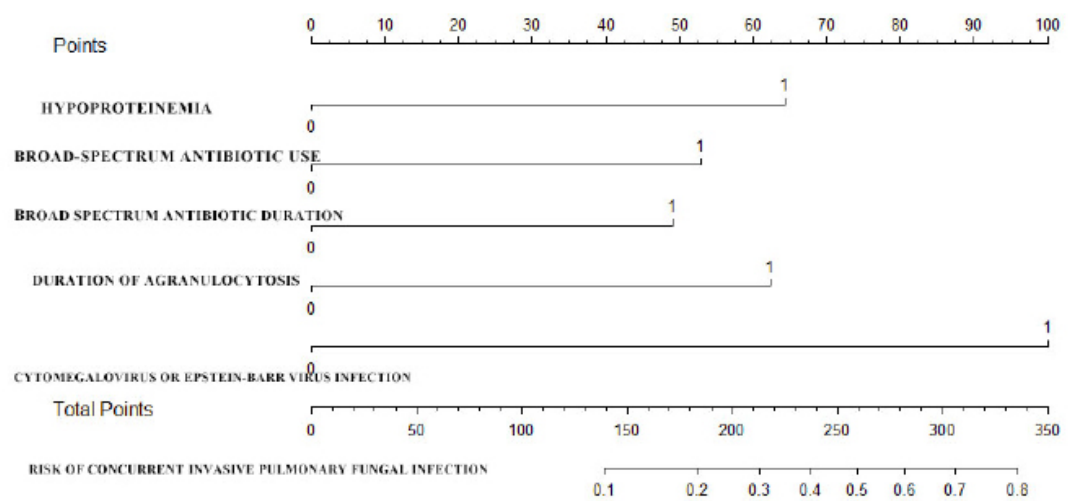

Fig. 1: Nomogram for IFD after chemotherapy in patients with hematologic tumors

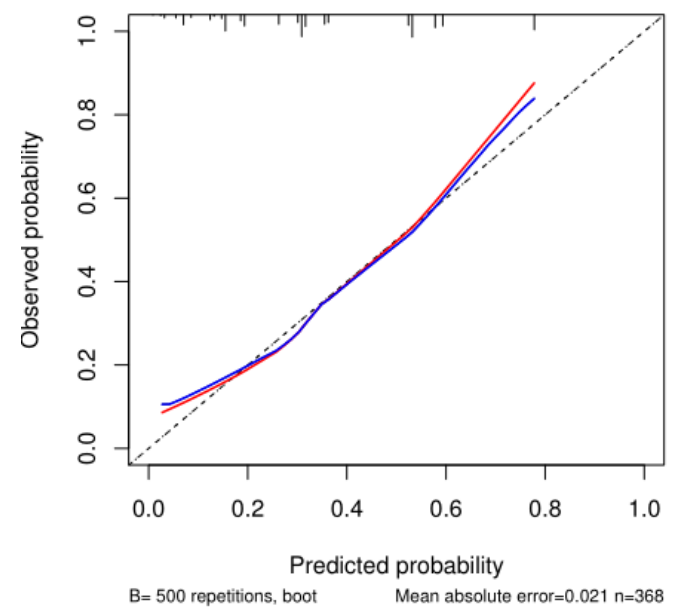

Fig. 2: Calibration curve verification for nomographic model, ( .... ) Ideal; ( -) Apparent; ( $\longrightarrow$ ) Bias-corrected

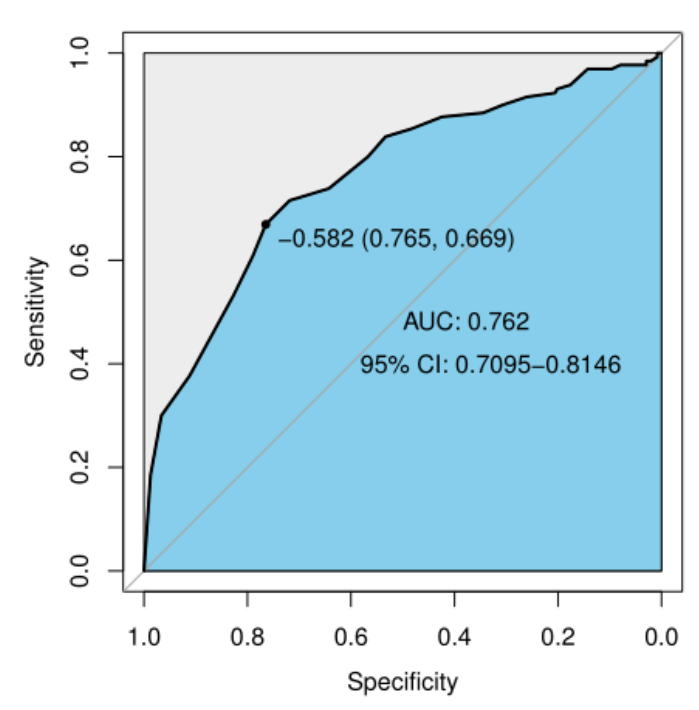

Fig. 3: ROC curve verification for nomographic model 
The occurrence and development of blood malignant tumor disease is closely related to the immune function defect of patients, one of which is that the patients with hematologic tumors have poor ability of self-resistance disease, accompanied by certain immune function damage; the other is whether they receive chemotherapy or radiotherapy, which will further damage the immune function of patients in the process of tumor treatment; the patients with hematologic tumors often have various malnutrition conditions. The combination of the three issues makes patients with hematologic tumors more susceptible to invasive fungal infections. It has been reported that co-occurrence of invasive fungal disease is one of the most important causes of mortality in patients with hematologic malignancies ${ }^{[7]}$. Nivoix et al. ${ }^{[8]}$ showed that the high mortality rate of fungal infection in patients with hematologic tumors may be related to other combined factors such as low clinical diagnosis, strong pathogenicity and co-infection with other bacteria. On the one hand, hematologic tumors and IFD may each exhibit different clinical manifestations due to different pathogenic fungal species and their infection sites. On the other hand, invasive fungal infection is most likely to involve the lungs, but due to cross-bacterial infection, disturbing the clinical diagnosis of invasive pulmonary fungal infection, it often missed the optimal treatment timing and the mortality rate of patients is very high. Therefore, exploring the risk factors of invasive pulmonary fungal infection in patients with hematologic tumors after chemotherapy can help to screen high-risk groups and give intervention measures in time, while establishing nomogram prediction model can reflect the situation of high-risk groups more intuitively and then taking individualized intervention, which has both theoretical and practical significance in reducing the incidence of infection, mortality and saving medical resources.

In this study, 368 patients after hematologic tumor chemotherapy were retrospectively analyzed. The incidence of complicated invasive pulmonary mycosis was $35.33 \%$. Multivariate analysis showed hypoproteinemia, hormone duration $>7 \mathrm{~d}$, broadspectrum antibiotics duration $\geq 7 \mathrm{~d}$, duration of agranulocytosis $>7 \mathrm{~d}$, cytomegalovirus or EpsteinBarr virus infections were the risk factors of IFD after chemotherapy for patients with hematologic tumors. The results were similar to those of Zhang ${ }^{[9]}$, who studied 375 cases of malignant blood disease complicated with invasive fungal infection in the Qinghai University Affiliated Hospital in Qinghai Province.
Analysis of risk factors-Malnutrition is a common complication in patients with malignant tumor. More than $50 \%$ of patients with malignant tumor have different degrees of hypoproteinemia. Hypoproteinemia can have adverse effects on various systems of the body, such as infection caused by decreased anti-inflammatory effect, edema caused by decreased osmotic pressure of colloid, increase of drug distribution volume and clearance caused by decreased ability to bind and transport substances, thus affecting the efficacy of drugs ${ }^{[10]}$. Protein deficiency affects not only its physical development and intellectual development, but also its abnormal physiological state, low immune function and reduced resistance to infectious diseases ${ }^{[11]}$. Therefore, the timely prevention and treatment of hypoproteinemia in patients with hematologic tumors due to chemotherapy should be based on adequate nutrition, increased supply of animal protein, vegetable protein and fresh vegetables, other symptomatic treatment and correction of hypoproteinemia. Fungi are conditionally pathogenic bacteria and usually do not cause infection. However, if antibiotics are used for a long time, especially the combination of two or more antibiotics, it may kill the normal strains, destroy the ecological balance of the normal flora of the host, lead to excessive growth of the fungi and become the dominant bacteria of the surface or cavity flora. In addition, the mucosal injury and granulocytopenia caused by chemotherapy and the weakened immune defense capacity inherent in the human body will eventually cause fungal infections ${ }^{[12,13]}$. On the one hand, patients with hematologic diseases often suffer from granulocytopenia, abnormal granulocyte phagocytosis and their defense function is reduced, which increases the risk of fungal infection. On the other hand, patients with hematologic tumors have a very long chemotherapy cycle and their long-term granulocytopenia or agranulocytosis after chemotherapy may gradually reduce their body immunity, which may eventually lead to complicated invasive fungal infection ${ }^{[14,15]}$. Persistent decreases in granulocytes have been reported to be associated with fungal infections, agranulocytosis lasting for $\geq 7 \mathrm{~d}$ and a significantly increased risk of fungal infections in the lung ${ }^{[16,17]}$. Therefore, patients with agranulocytosis should be actively treated with targeted therapy to improve the value. The autoimmune function of patients with hematologic tumors is poor, the immune function is further decreased after chemotherapy and it is more likely to be complicated with infection. Cytomegalovirus is a disease-causing virus that lurks in body for long periods of time, waits 
for time and increases infection risk of other pathogens including bacteria, fungi and other viral infections through affecting immune system function ${ }^{[18]}$; EpsteinBarr virus is a lymphofollicular virus that activates infected or transfected B cells and Epstein-Barr virus increases the risk of certain cancers and immune system diseases and is associated with multiple autoimmune diseases and the disease secondary to infection is of great importance to clinicians.

In this study, 500 times of repeated sampling by the Bootstrap method were used to verify internally based on the multivariate logistic regression analysis. The calibration curve fitted well with the standard curve. The ROC curve AUC was 0.762, $95 \%$ CI: 0.7095 0.8146 and the Hosmer-Lemeshow test $p=0.200>0.05$, suggesting that the nomogram prediction model has good discrimination and accuracy, and it has high clinical value. The clinical medical staff shall attach great importance to the high-risk population, give targeted and effective intervention measures, and reduce the risk of invasive pulmonary fungal infection induced by various risk factors as far as possible, such as providing adequate nutrition for prevention and treatment of hypoproteinemia, increasing the supply of animal protein, vegetable protein and fresh vegetables, monitoring and correcting water, electrolyte disorders and skin care; strictly reducing the dosage of controlled hormone and drug withdrawal, and timely supplementing calcium element; correcting use of hormones and broad-spectrum antibiotics, close monitoring of patients with agranulocytosis, etc.

In conclusion, hypoproteinemia, broad-spectrum antibiotic use $\geq 2$ kinds, broad-spectrum antibiotic duration $\geq 7 \mathrm{~d}$, duration of agranulocytosis $\geq 7 \mathrm{~d}$ and cytomegalovirus or Epstein-Barr virus infection are the risk factors of concurrent IFD after chemotherapy in patients with hematologic tumor. After verification, the constructed nomogram has high discrimination and accuracy, which has dual guiding significance for clinical screening of high-risk population and making individualized prevention and treatment measures.

\section{Conflict of interests:}

The authors declared no conflict of interest.

\section{REFERENCES}

1. Lei XW, Han Y. Research progresses in immunotherapy of hematologic neoplasms. Int J Blood Transfus Hematol 2020;43(2):175-80.

2. Hu D, Ding L, Huang WQ, Du QR, Liu B, Yu Y. Risk factors of occurrence of adverse events in hematological malignancies after chemotherapy. J Clin Med Pract 2020;24 (2):55-7.
3. Huang Y, Wu S, Zhang Y, Zi YM, Yang M, Guo Y, et al. Clinical features and therapeutic measures of patients with hematologic malignancies complicated by pulmonary fungal infections during neutropenic phase after chemotherapy. Chin J Nosocomiol 2016;26(7):1536-8.

4. $\mathrm{Gu} \mathrm{X}, \mathrm{Xu} \mathrm{S}, \mathrm{Liu} \mathrm{GM}$, Wan QF. Risk factors, clinical manifestations and prognosis of invasive pulmonary fungal infection. J Clin Pulm Med 2015;20(11):2090-3.

5. Zhang Z, Shen T. Criteria for diagnosis and treatment of hematologic diseases. 3rd edn. Beijing: People's Medical Publishing House; 2007. p. 106-235.

6. Editorial committee of Chinese journal of internal medicine. Diagnostic criteria and therapeutic principles for invasive fungal infections in patients with hematologic diseases/ malignancies. Chin J Intern Med 2005;44 (7):554-6.

7. Neofytos D, Horn D, Anaissie E, Steinbach W, Olyaei A, Fishman J, et al. Epidemiology and outcome of invasive fungal infection in adult hematopoietic stem cell transplant recipients: Analysis of multicenter Prospective Antifungal Therapy (PATH) alliance registry. Clin Infect Dis 2009;48(3):265-73.

8. Nivoix Y, Velten M, Letscher-Bru V, Moghaddam A, NatarajanAmé S, Fohrer C, et al. Factors associated with overall and attributable mortality in invasive aspergillosis. Clin Infect Dis 2008;47(9):1176-84.

9. Zhang L. Characteristics of invasive fungal infections in 375 patients with hematologic malignancies. Qinghai University; 2018.

10. Zhou G, Yang D. Research progress in hypoproteinemia. Guizhou Med J 2015(3):279-81.

11. Sun $\mathrm{C}$, Guo X, Xu XH, Wang L. Research progress on the relationship between hypoproteinemia and clinical outcome of various diseases. J Chengde Med Coll 2015;32(5):435-7.

12. Liu D, Wang Y, Zheng R. Clinical analysis of invasive pulmonary fungal infection during agranulocytosis in malignant hematologic diseases. Front Med 2013;13(13):15960.

13. Lehrnbecher TA, Frank C, Engels K, Kriener S, Groll AH, Schwabe D. Trends in the postmortem epidemiology of invasive fungal infections at a university hospital. J Infect 2010;61(3):259-65.

14. Signe J, Jung B, Nougaret S, Belafia F, Panaro F, Bismuth M, et al. Pneumonia in immunocompromised patients: More than meets the eye. Am J Respir Crit Care Med 2012;186(11):e18.

15. Chen W, Xu YH. Statistical analysis on the hospitalized patients of invasive fungal infection concurrent with hematological malignancies. Chin Med Rec 2017;18(1):101-3.

16. Zhang H, Long B. Clinical characteristics and risk factors of infection after chemotherapy for acute leukemia. Chin J Mod Med 2018;20(1):38-40.

17. Cao LF, Xu A. Progress in diagnosis of invasive pulmonary fungal infection. J Clin Pulm Med 2011;16(11):1750-2.

18. Chen H. Great importance should be attached to the harm of cytomegalovirus infection after liver transplantation. J Pract Organ Transplan 2021;9(1):17-20.

This is an open access article distributed under the terms of the Creative Commons Attribution-NonCommercial-ShareAlike 3.0 License, which allows others to remix, tweak, and build upon the work non-commercially, as long as the author is credited and the new creations are licensed under the identical terms

This article was originally published in a special issue, "Novel Therapeutic Approaches in Biomedicine and Pharmaceutical Sciences" Indian J Pharm Sci 2021:83(6) Spl Issue "110-117" 